\title{
Large Artery Stiffness Assessment Using SphygmoCor Technology
}

\author{
Mark Butlin ${ }^{a} \quad$ Ahmad Qasem ${ }^{a, b}$ \\ a Department of Biomedical Sciences, Faculty of Medical and Health Sciences, \\ Macquarie University, and ${ }^{\mathrm{b}}$ AtCor Medical, Sydney, NSW, Australia
}

\section{Keywords}

Large artery stiffness · SphygmoCor technology

\begin{abstract}
Large artery stiffness assessment has been an integral part of the SphygmoCor technology since 1998. Aortic stiffness is approximated with non-invasive measurement of carotid-femoral pulse wave velocity, with improvements made with time to make the assessment procedure quicker and more user independent. Also standard in the devices is the ability to reliably calculate the central aortic waveform shape from a peripheral pressure waveform from either the brachial or radial artery. This waveform contains much information beyond peak and trough (systolic and diastolic pressure). Relative waveform features such as the augmentation index, wave reflection magnitude, reflection time index, and subendocardial viability ratio are parameters that are influenced by the stiffness of systemic arteries. This article briefly describes these parameters related to large artery stiffness and provides reference to validation and repeatability studies relative to the clinical use of the SphygmoCor devices. It is beyond the scope to review here the 424 original research articles that have employed SphygmoCor devices in measuring arterial stiffness. Instead, the method of measurement across the devices is described, including tonometry, volumetric displacement through cuff placement around limbs, and ambulatory monitoring. Key population and subpopulation studies are cited where the average stiffness parameter progression with age and gender, as measured by SphygmoCor devices, is quantified in the healthy and general population. Finally, with reference to guidelines from working groups on arterial stiffness and hypertension, the clinical utility of large artery stiffness measurement is discussed in the context of the arterial stiffness parameters provided by the SphygmoCor systems.




\section{Introduction}

The stiffness of the systemic large arteries more than doubles with age [1], is associated with outcome in many different cardiovascular diseases [2], and is a recommended parameter to measure in the management of hypertension [3]. Stiffening of the large arteries increases the speed of the ejected pulse from the left ventricle through the arteries and results in an earlier return of the reflected pressure. This can occur to an extent that it augments pressure during the period of left ventricular ejection. The early arrival of the reflected pulse during systole increases the afterload on the left ventricle and reduces coronary artery perfusion pressure during diastole. This risk of increased arterial stiffness is seen in measures of large artery stiffness being independently predictive of coronary artery disease, stroke, and cardiovascular events in general [4].

Much of the research into the importance of arterial stiffness in disease progression has been through non-invasive analysis of the pulse wave velocity (PWV) through the large arteries. Waveform shape parameters of the central, aortic blood pressure waveform are also related to arterial stiffness, and non-invasive reconstruction of the aortic blood pressure waveform has permitted inclusion of these parameters in studies as well. One of the earliest devices in the field for non-invasive PWV and aortic pressure waveform assessment was the SphygmoCor Cardiovascular Management Suite (CvMS; AtCor Medical, Sydney, NSW, Australia). This device relied on applanation tonometry for acquisition of the radial, carotid, and femoral blood pressure waveforms. The SphygmoCor technology has more recently been applied to volumetric displacement (cuff-based) acquisition of pulse information (SphygmoCor XCEL). The cuff-based approach means that the arterial stiffness assessment requires less user expertise and makes the process less operator dependent. It has also allowed the technique to be applied to ambulatory blood pressure devices for analysis of out-of-clinic blood pressure values and variability throughout the day and night (Oscar 2 with SphygmoCor Inside; SunTech Medical, Morrisville, NC, USA). This article describes the methodology, validation, and clinical use of these devices in assessing arterial stiffness.

\section{Technical Aspects}

\section{Acquisition of Peripheral Pressure Waveform Signals}

Both the SphygmoCor CvMS and SphygmoCor XCEL devices (Fig. 1) measure the same parameters related to the central aortic pressure waveform parameters and carotid-femoral arterial PWV. The main difference lies in the different method of peripheral arterial waveform acquisition with the XCEL (brachial and femoral cuff-based acquisition), addressing several limitations of the CvMS (radial and femoral artery tonometry) method of peripheral waveform acquisition. The limitations of tonometry in acquiring a peripheral waveform are: (1) difficulties in obtaining a high-quality radial or femoral pulse in some subjects with lower blood pressure, with a more difficult pulse to palpate, or with obesity; (2) operator dependency of the peripheral signal acquisition; and (3) calibrating the radial arterial pressure signal by equating radial arterial systolic and diastolic peaks with systolic and diastolic blood pressure at a nearby (brachial) site, given the difference between radial and brachial arterial blood pressure is small.

These issues are addressed in the SphygmoCor XCEL device, which uses a conventional brachial cuff to measure systolic and diastolic pressure and then acquires a volumetric displacement signal from the same cuff inflated to a sub-diastolic level of pressure. In the case of PWV assessment, the XCEL device uses the volumetric displacement waveform from a cuff around the upper thigh in place of femoral artery tonometry. The method is robust in all 
Fig. 1. a, b The SphygmoCor CvMS device, which utilizes tonometry (b) for acquisition of the peripheral vascular waveform. c, d The SphygmoCor XCEL device, which utilizes cuff-based volumetric displacement (d) for acquisition of the peripheral vascular waveform.
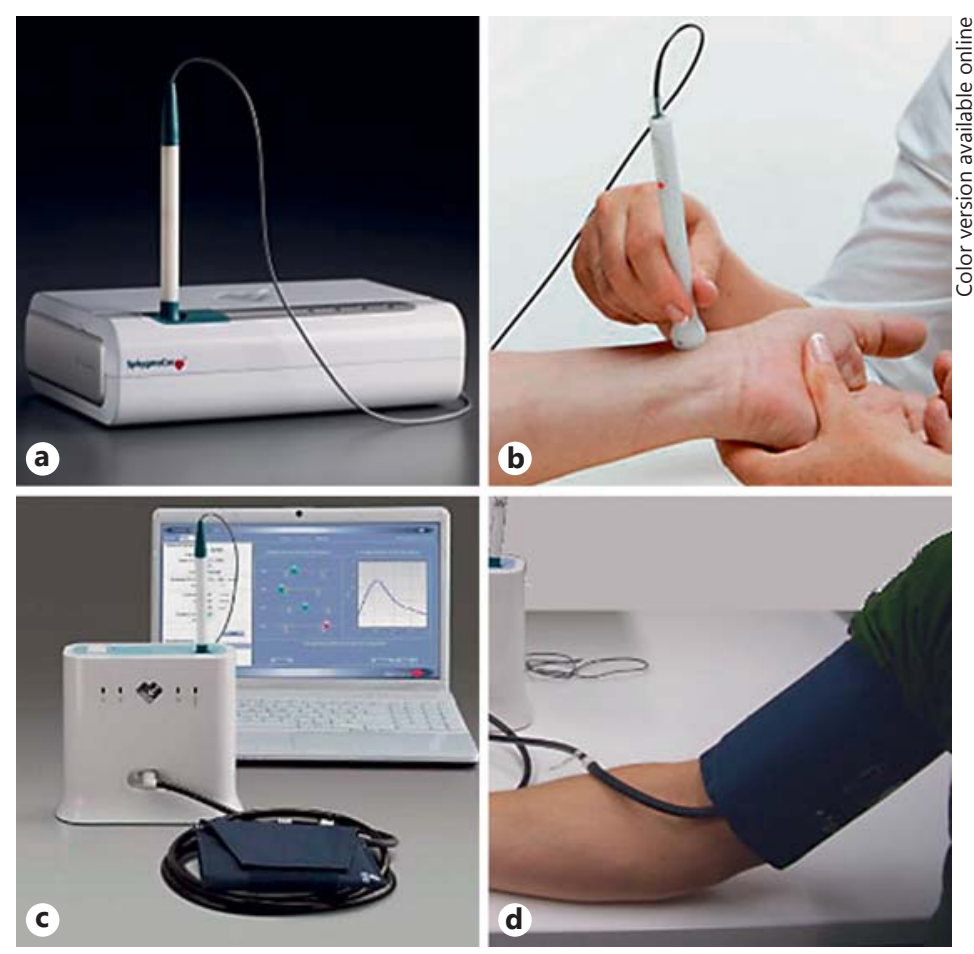

subjects, including those with low blood pressure or with a hard-to-palpate pulse. The method is user independent; it requires the user to place the cuff on the subject's upper arm (or leg) and press a button to take the measurement. In the case of PWV assessment, tonometry is still used in the XCEL device for acquisition of the carotid pulse. The method also addresses the calibration issue, by calibrating the brachial signal with the measured brachial arterial pressure. The acquisition of the femoral pulse by cuff for carotid-femoral PWV (cfPWV) assessment also removes the need for an electrocardiogram to align sequentially acquired signals, as employed in the SphygmoCor CvMS. Whilst there is a strong move toward cuffbased acquisition for its ease of use in the clinical setting, tonometry offers other advantages. Namely, tonometry acquisition of the peripheral pulse captures much higher-frequency information than that obtained by cuff volumetric displacement, which inherently dampens higher-frequency information. Thus, tonometry still has a place in research applications where high-frequency components of the waveform are of interest.

\section{Carotid-Femoral Pulse Wave Velocity}

Aortic PWV is related to the intrinsic material stiffness of the aorta and is a major determinant of the pressure load on the heart both through the compliance of the aorta itself and the transmission of the forward and reflected pressure wave. Aortic PWV cannot be measured directly by non-invasive means, with the exception of expensive, non-portable techniques such as phase contrast magnetic resonance imaging (MRI) [5]. Instead, other arterial sites are used to provide a PWV surrogate for aortic PWV. The 2 most common arterial sites are the carotid and the femoral arteries, as they are non-invasively accessible and bridge the aortic path length. Both the SphygmoCor CvMS and XCEL measure cfPWV, but with 2 different methodologies.

The SphygmoCor CvMS appeared on the market in 2008 and has been used in a multitude of population studies since. The carotid and femoral pulse is acquired by applanation 
Fig. 2. SphygmoCor CvMS carotidfemoral pulse wave velocity (cfPWV) measurement method with sequential applanation tonometry of the carotid (c) and femoral (f) sites. Pulse transit times (tt) are calculated from the electrocardiogram (ECG) R-wave to the foot of the applanated waves. Distances (d) are measured from the suprasternal notch (s) to the sites of applanation tonometry.

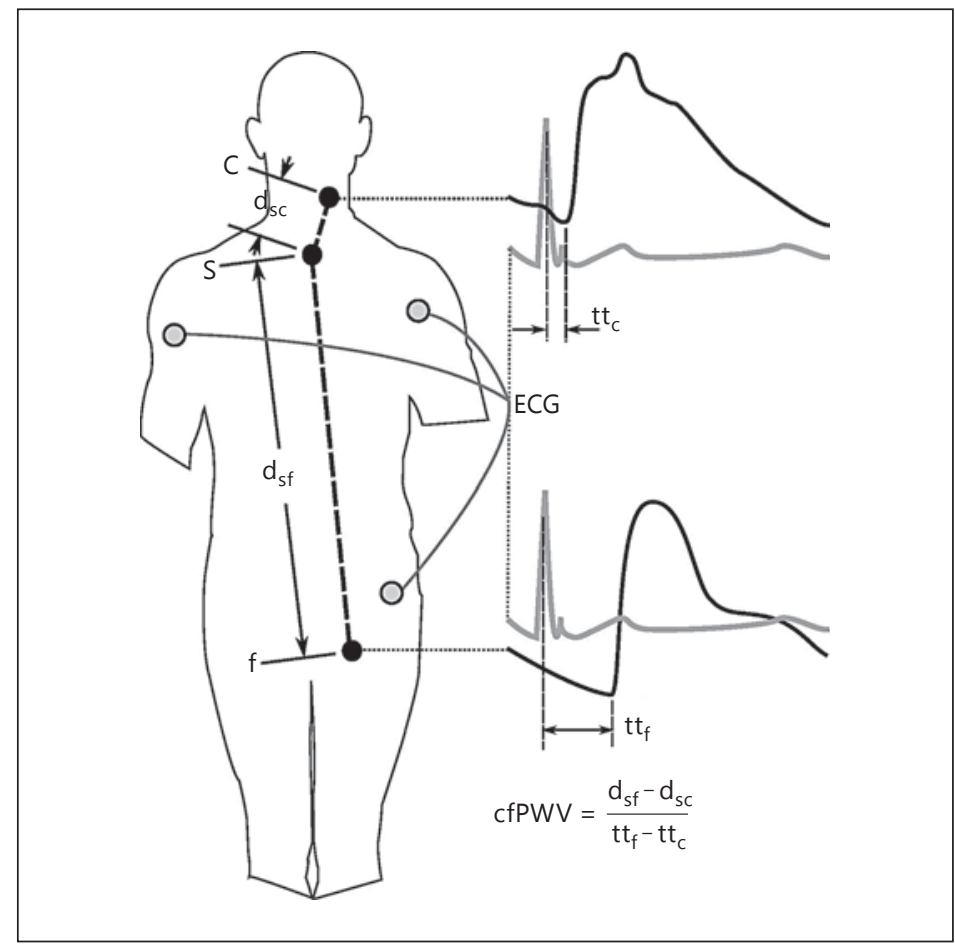

tonometry sequentially, allowing a single operator to acquire the measurement. The transit time from the R-wave of the simultaneously acquired electrocardiogram to the foot of the carotid and femoral pulse is measured. The difference between these 2 transit times is divided by distances measured from the body surface to estimate the arterial path length in order to calculate the cfPWV (Fig. 2). The SphygmoCor cfPWV had been established in the literature as a reference for comparison due to the wealth of population data and healthy population reference values [6]. It is recommended in the ARTERY Society guidelines for the validation of PWV devices as the "secondary standard" that new PWV devices be validated against [7].

Measurement of cfPWV using the SphygmoCor CvMS is reproducible with a withinobserver variability of $0.07 \pm 1.17 \mathrm{~m} / \mathrm{s}$ and a between-observer variability of $0.30 \pm 1.25 \mathrm{~m} / \mathrm{s}$ in healthy subjects [8], as well as with an inter-observer difference of $0.3 \pm 3.2 \mathrm{~m} / \mathrm{s}$ in chronic kidney disease patients [9]. The measure has been validated against invasive measurement of aortic PWV from the ascending aorta to the iliac bifurcation in 135 people, with noninvasive cfPWV having a mean difference from invasive aortic $\mathrm{PWV}$ of $-0.2 \mathrm{~m} / \mathrm{s}$ for an average aortic PWV of $8.5 \pm 2.4 \mathrm{~m} / \mathrm{s}$ [10], a $2.4 \%$ deviation from the invasive value. The non-invasive measure of cfPWV has also been compared to PWV assessed across a similar aortic path length measured using phase contrast MRI in a cohort of 162 subjects, with the mean difference between the measures being $1.7 \pm 1.6 \mathrm{~m} / \mathrm{s}$ for a mean cfPWV of $7.1 \pm 1.4 \mathrm{~m} / \mathrm{s}$ [5].

The vascular path length is estimated by measuring linear distances between surface points on the body (Fig. 2). As the calculation of the transit time involves subtraction of the transit times of the pulse travelling in opposite directions, it is logical to subtract the distances from central to peripheral points. Comparison to path lengths measured using MRI shows that the subtraction technique underestimates the vascular path length by, on average, $0.05 \pm 0.04 \mathrm{~m}$ [11], where the average path length is around $0.5 \mathrm{~m}$. Whilst other methods of distance measurement have been proposed that decrease the error slightly, the subtraction of suprasternal notch-to-carotid distance from the suprasternal notch-to- 


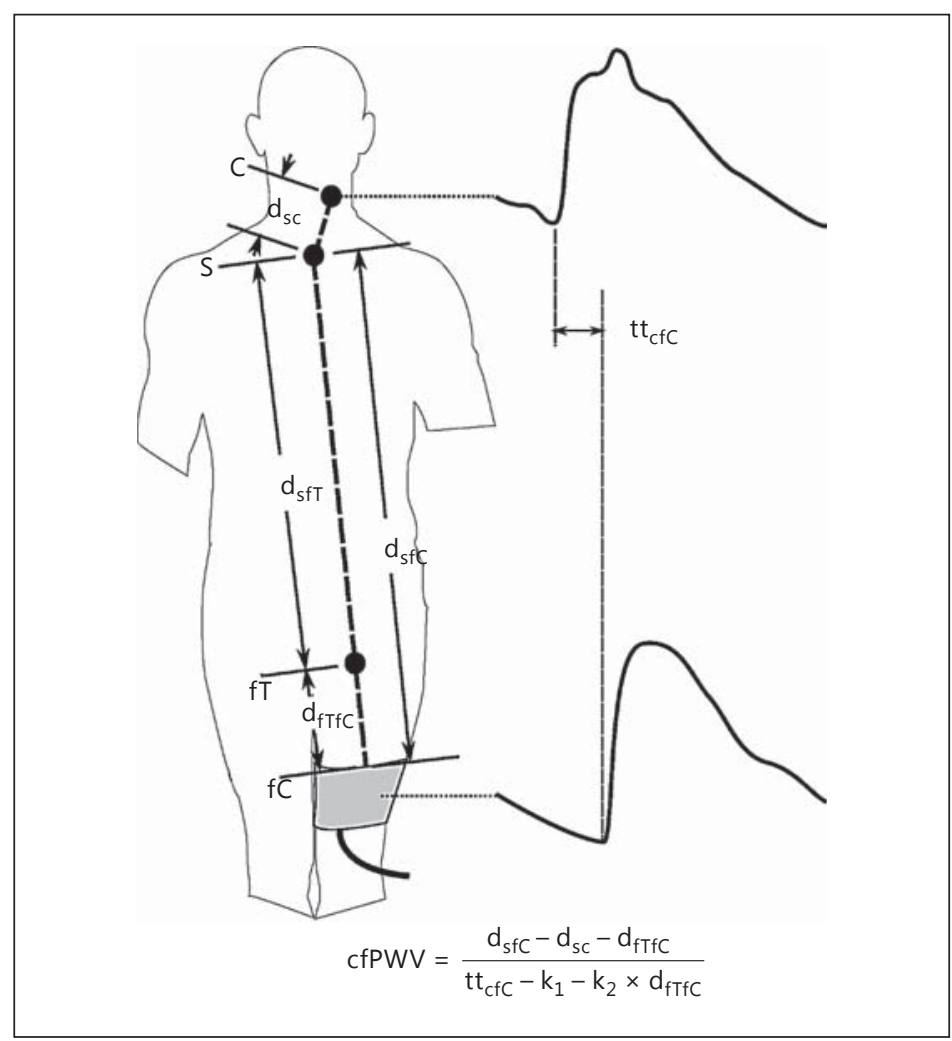

Fig. 3. SphygmoCor XCEL carotid-femoral pulse wave velocity (cfPWV) measurement simultaneously acquires a carotid (c) pulse by applanation tonometry and a femoral (f) pulse by volumetric displacement within a cuff $(\mathrm{C})$ around the upper thigh and measures the transit time $(\mathrm{tt})$ between the feet of the 2 waves. Distances (d) are measured from the suprasternal notch (s) to the top of the thigh cuff (fC), the site of carotid tonometry (c), and to the site where the femoral artery could be applanated by tonometry (fT). cfPWV is calculated equivalent to the SphygmoCor CvMS calculation (Fig. 2) by subtracting the contribution of the additional femoral segment to both the distance $\left(\mathrm{d}_{\mathrm{fTfC}}\right)$ and the transit time proportional $\left(\mathrm{k}_{2}\right)$ to that distance. A further correction to the transit time $\left(\mathrm{k}_{1}\right)$ is made to adjust for the delay in transmission of the pulse from the femoral cuff to the pressure transducer, as opposed to the carotid tonometer where the transducer is placed on the skin above the artery.

femoral distance is the method that has been used to establish population normal values of cfPWV $[6,12]$ and has been shown to have a $0.2 \mathrm{~m} / \mathrm{s}$ difference from invasively measured aortic PWV [10].

Even though the SphygmoCor PWV device is considered a standard tool for assessing arterial stiffness, the measurement procedure is not ideal for clinical practice. Firstly, the sequential pulse acquisition is time-consuming and relies on minimal changes in blood pressure and heart rate during the measurement for an accurate PWV assessment. Secondly, the acquisition of a good femoral artery pulse by applanation tonometry in some patients requires a high level of operator skill. Thirdly, the acquisition of the femoral pulse requires some clothing to be removed for the femoral site to be applanated, making the measurement intrusive. The SphygmoCor XCEL device, introduced into the market in 2012, addresses these problems through acquisition of the femoral pulse by volumetric displacement within a cuff placed around the upper thigh (Fig. 3). This removes the need for applanation of the femoral pulse and allows for simultaneous acquisition of the carotid and femoral pulse. 
The cfPWV can be calculated by measuring the foot-to-foot transit time between the carotid and cuff-acquired femoral pulse and dividing it by a measured distance. However, this cfPWV will have a different magnitude than the cfPWV assessed using the SphygmoCor CvMS (based on which population normal values have been established) because of the inclusion of an additional femoral artery segment; this is due to femoral artery stiffness changing at a different rate to aortic stiffness with age [13]. Therefore, the contribution of the additional femoral segment to the pulse transit time is subtracted using population normal values to arrive at a cfPWV equivalent to that obtained if it were measured using the SphygmoCor CvMS [14].

The correction for the additional femoral segment requires measuring the distance from the top of the thigh cuff to the site where the femoral artery would have been applanated and subtracting this distance - and the calculated transit time for the pulse to travel this distance - from the calculation of cfPWV (Fig. 3).

The SphygmoCor XCEL measure of cfPWV has been validated [14] as per the ARTERY PWV validation guidelines [7]. The SphygmoCor XCEL device was compared to the SphygmoCor CvMS for measurement of cfPWV in 90 subjects with an average error ( \pm SD) of $0.01 \pm 0.71 \mathrm{~m} / \mathrm{s}$ with a correlation of 0.93 [14]. According to the ARTERY Society guidelines, the accuracy of the XCEL cfPWV is "excellent" (mean difference $<0.5 \mathrm{~m} / \mathrm{s}$, SD of difference $<0.8 \mathrm{~m} / \mathrm{s}$ ).

\section{Pressure Wave Reflection}

The incident - or forward-going - pressure wave in the aortic arch is reflected from sites of impedance mismatch such as arterial branching, changes in arterial diameter, and changes in vessel wall material stiffness. The magnitude of the reflected waveform is therefore dependent upon the geometry and the stiffness of the arterial tree distal to the aortic arch. Therefore, parameters of the aortic pressure waveform that relate to wave reflection are influenced by the stiffness of the distal arterial tree. Non-invasive measurement of the aortic waveform morphology allows these parameters to be quantified in both a clinical environment and in population studies.

The SphygmoCor systems integrate pressure pulse wave analysis, developed from the concept that there is haemodynamic information contained in the shape of the arterial pressure pulse that can be used to supplement the conventional measurement of blood pressure. The systolic and diastolic values of blood pressure are the maximum and minimum points of the pressure curve obtained in a peripheral location, usually the upper arm. However, similar values of systolic and diastolic pressures can be associated with many different pulse wave shapes (Fig. 4), and these determine the type of interaction between the heart as a pump and the arterial system as the load.

One of the main attributes of the SphygmoCor systems is the ability to derive the central aortic pressure waveform non-invasively from the pressure pulse recorded at a peripheral site. Two specific things made this possible and contributed to the practical use of the device in both research and clinical situations: (1) the ability to accurately record the peripheral pulse and (2) a common relationship between the frequency components of the aortic and peripheral pressure waveform shapes across the adult population, quantified as a generalized transfer function [15].

The SphygmoCor CvMS detects radial arterial pressure through applanation tonometry, while the SphygmoCor XCEL records the volumetric displacement related to the volume of the brachial artery within a cuff inflated around the upper arm. A general transfer function is applied to the non-invasively acquired peripheral signal to calculate the aortic waveform. The SphygmoCor systems calculate over 20 different parameters quantifying the aortic waveform. Related to the stiffness of large arteries are the reflection time index, the reflection magnitude, 
Fig. 4. Peripheral (radial) and aortic arterial blood pressure waveforms from 2 sample patients. Whilst "Jan" (solid line) and "Joe" (dashed line) both have a systolic/diastolic blood pressure in the arm of $140 / 80 \mathrm{~mm} \mathrm{Hg}$, the aortic blood pressure magnitude is markedly different, with "Joe" having a blood pressure of 136/80 mm Hg and "Jan" having a blood pressure of 115/82 mm Hg. This difference in magnitude is relayed in the waveform shape of the peripheral waveform.

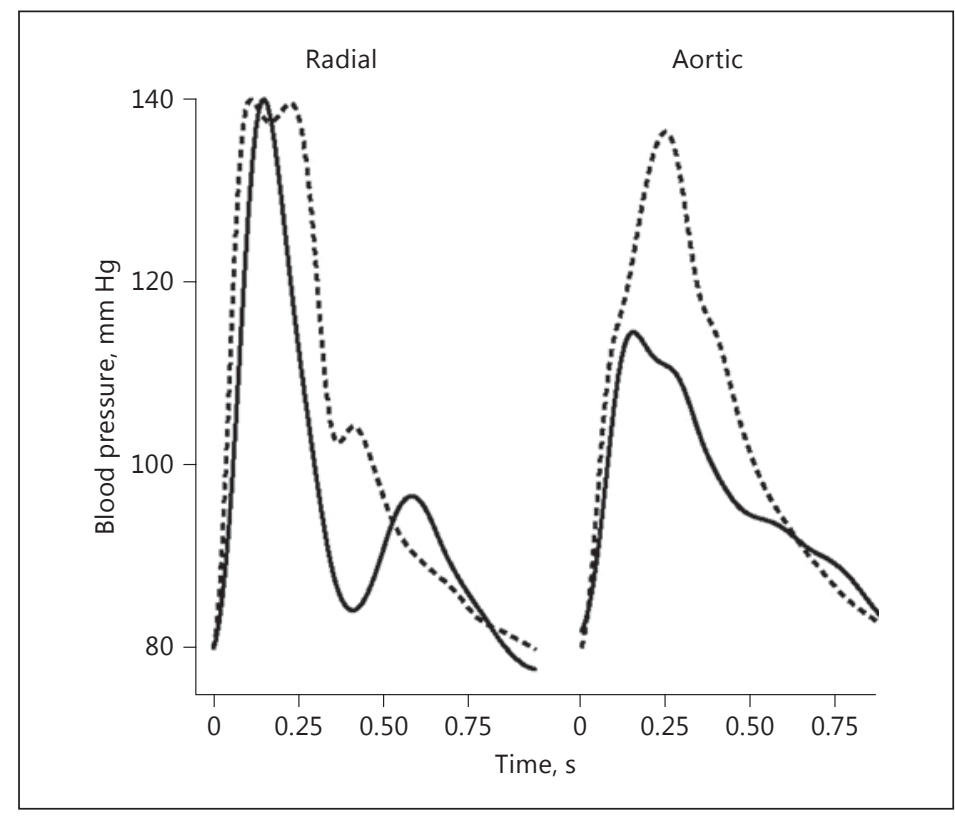

and the aortic augmentation index (AIx). The augmentation of the pressure wave also has implications for the relative pressure during systole and diastole, summarized in the subendocardial viability ratio (SEVR).

\section{Augmentation Index}

The AIx is a measure of the degree to which the peak of a measured pressure wave is over and above the peak of the incident pressure wave due to the addition of the reflected pressure wave. The AIx is dependent on the timing and magnitude of the reflected waveform and is influenced by the compliance and structure of vessels distal to the site of measurement.

Whilst 2 methods of calculating the AIx exist [1], the SphygmoCor systems use the more common expression of AIx as the augmentation pressure (systolic pressure minus the inflection pressure; Fig. 5) divided by the pulse pressure (systolic minus diastolic pressure), expressed as a percentage.

As the AIx is dependent on the transit time of the reflected wave and the time of arrival of the reflected wave during the pressure pulse, it is also sensitive to heart rate. A slower heart rate will cause the reflected wave peak to occur relatively earlier in systole, which will increase the AIx. Conversely, a faster heart rate is associated with the reflected wave arriving relatively later in systole, or during diastole, causing a decrease in the AIx. The SphygmoCor software reports the AIx at the patient's current heart rate, as well as giving an AIx corrected to a heart rate of $75 \mathrm{bpm}$ using a regression to the population heart rate dependency of the AIx [16]. The AIx at a heart rate of $75 \mathrm{bpm}$ is a useful parameter in studies where the average heart rate is different between groups or individuals, and thus the AIx alone cannot be used in statistical models without inclusion of heart rate.

Reliable measurement of the aortic AIx is dependent only on waveform shape, not magnitude, as it is expressed as a proportion of pulse height. Therefore, calculation of the aortic AIx is dependent upon accurate prediction of the aortic pressure waveform shape from the peripheral waveform, using the general transfer function [15]. Measurement of the aortic AIx from the tonometrically acquired radial pulse (Colin JENTOW 7000 tonometer system) with application of the same general transfer function as applied in the SphygmoCor system 
Fig. 5. Example of an aortic pressure waveform of a nominal pulse height from diastolic pressure (DP) to systolic pressure (SP). The anacrotic shoulder is associated with the commencement of augmentation of the forward-going pressure wave by the reflected pressure wave. The pressure at which this occurs is the inflection pressure $\left(\mathrm{P}_{\mathrm{i}}\right)$. The incisura, not to be confused with the dicrotic notch seen in more peripheral waves, is associated with the closing of the aortic valve and the end of systole $\left(\mathrm{P}_{\mathrm{es}}\right)$. The dotted line shows an estimated flow wave of arbitrary units using these points as a guide for the waveform shape. The subendocardial viability ratio can be calculated as the ratio of systolic area to diastolic area.

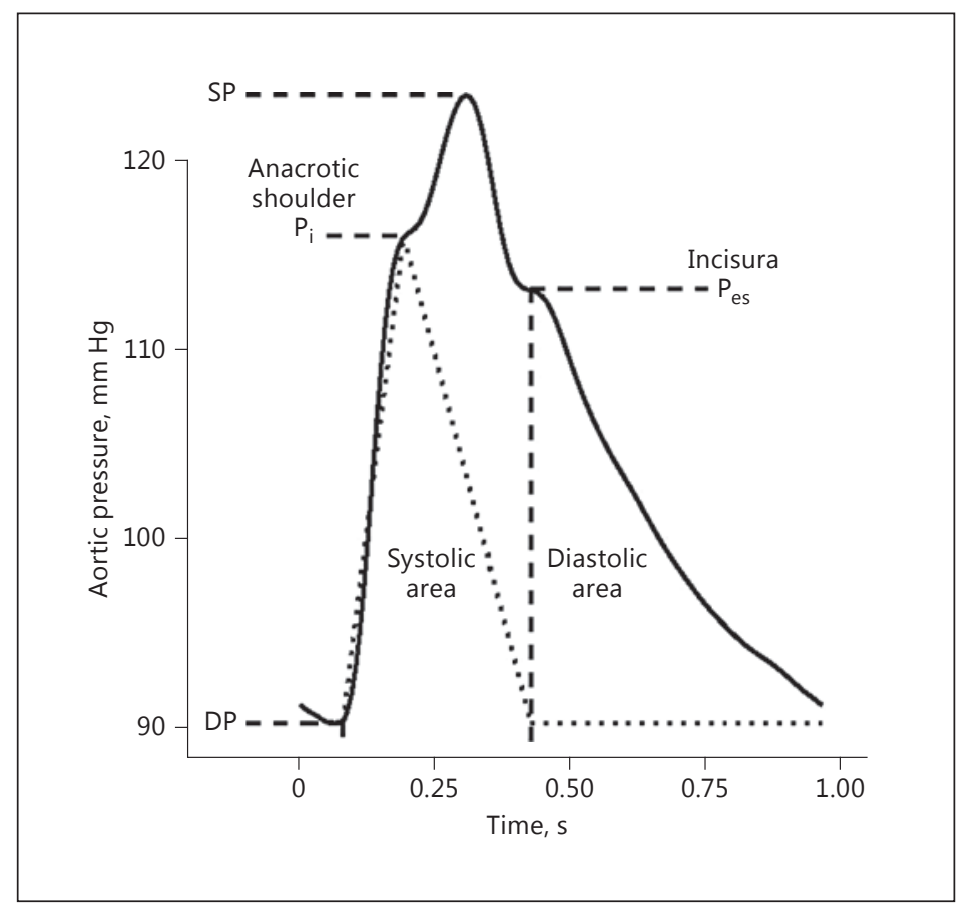

has been validated against the aortic AIx being calculated from invasively measured pressure in both the resting condition [17] and during forced blood pressure changes through a Valsalva manoeuver [18], with a difference between invasive and derived methods of calculating the AIx of $-7 \pm 9 \%$. Using the SphygmoCor CvMS specifically, aortic AIx from the calculated aortic waveform has been compared against invasive measures during rest and the Valsalva manoeuver [19], and the augmentation pressure itself measured during intravenous nitroglycerine administration (difference from invasively measured value of $1 \pm 8 \mathrm{~mm} \mathrm{Hg}$ ) [20]. AIx measured using the SphygmoCor XCEL has been compared to that measured with the SphygmoCor CvMS, with a non-significant mean difference of $1.6 \pm 2.0 \%$ [21].

A number of studies have quantified the reproducibility of aortic AIx measured using the SphygmoCor CvMS system. In healthy subjects of cohort sizes between 25 and 88, the withinobserver difference varied from $0.5 \pm 5.4$ to $1.4 \pm 1.2 \%$, and the inter-observer difference varied from $0.2 \pm 3.8$ to $1.5 \pm 1.3 \%[8,22,23]$. In patients with cardiogenic shock, withinobserver repeatability was $0.1 \pm 5.8 \%$ [24], and in chronic renal failure patients, withinobserver repeatability was $0 \pm 4 \%$ and inter-observer repeatability was $0 \pm 3 \%$ [25].

\section{Reflection Magnitude}

The reflection magnitude is expressed as the amplitude of the reflected wave over the amplitude of the forward-travelling wave. The reflection magnitude can be calculated by resolving the incident and reflected waves from the measured pressure waveform. The advantage of calculating the reflection magnitude in place of the AIx is that the reflection magnitude is not heart rate dependent - as, unlike the AIx, it is independent of the timing of the inflection point. However, resolving the incident and reflected pressure waves for calculation of the reflection magnitude requires both a measured pressure and a flow waveform at the same site, which are then decomposed into their frequency components [26].

The SphygmoCor systems calculate aortic pressure from a peripheral waveform. For the purposes of calculating the reflected wave magnitude, aortic flow can be approximated by a 
triangular waveform that is zero at diastole and the end of systole, and peaks at the anacrotic shoulder (Fig. 5) [27]. This method is employed in the SphygmoCor systems and provides a non-invasive way of estimating the reflection magnitude. This procedure was validated using invasive pressure and flow recording and showed approximately a 0.1 difference between the reflection magnitude calculated using the estimated flow wave and that calculated using the measured flow wave [27].

Reflection Time Index

Related to the aortic PWV, the reflected pressure wave returns to the origin faster in a stiffer arterial system. The reflection time index is the time from beginning of systole to the arrival of the reflected wave expressed as a fraction of the cardiac period. The timing of the arrival of the reflected wave is located approximately at the inflection in the pressure waveform, the anacrotic shoulder (Fig. 5). The SphygmoCor systems decompose the pressure waveform into the forward and backward components, as outlined in the previous section, using a triangular estimate of aortic blood flow along with the derived pressure to calculate impedance. The resulting reflection time has a high correlation with the cfPWV [28], indicating that it is also a marker of large artery stiffness.

Measurement of the time to the reflected wave has an intra-observer repeatability of $0 \pm$ $20 \mathrm{~ms}$ and an inter-observer repeatability of $1 \pm 7 \mathrm{~ms}$ [25]. The reflection time index itself has a within-observer repeatability of $0.14 \pm 1.2 \%$ in subjects with cardiogenic shock [24] and a $0.4 \pm 5.8 \%$ within-observer and $1.6 \pm 2.3 \%$ inter-observer repeatability in a healthy population [22].

Subendocardial Viability Ratio

The shape of the aortic pressure pulse is a result of the ventricular ejection and the physical properties of the arterial system. The load on the ventricle during ejection is described by the pressure during systole. The contracting ventricle of a normal heart is able to eject blood under a range of pressure loads, so even if the pressure is changed, the form of the ejection wave is quite similar. If wave reflection occurs during systole, it will increase the pressure against which the ventricle has to eject its contents. Thus, in addition to having the values of systolic and diastolic pressure in the aorta, knowledge of the pressure waveform will facilitate analysis of the coupling between the ejecting heart and the pressure load. The SEVR, also known as the Buckberg index, is the area under the pressure curve during diastole as a ratio of the area under the pressure curve during systole (Fig. 5). The SphygmoCor systems report this parameter with the end of systole being determined by the incisura of the aortic pressure waveform (Fig. 5).

Repeatability studies for the SEVR measured using the SphygmoCor CvMS have been conducted in healthy subjects [23], chronic kidney disease patients [9], renal failure patients [25], and hypertensives [29], with intra-observer differences ranging from $0 \pm 18$ to $2 \pm 39 \%$, and inter-observer repeatability ranging from $1 \pm 19$ to $3 \pm 15 \%$.

\section{Ambulatory Blood Pressure Monitoring}

The Oscar 2 device, manufactured by SunTech Medical, is an ambulatory blood pressure monitor that has recently been upgraded to include the same algorithm as used in the SphygmoCor XCEL, using a similar protocol but in an ambulatory setting. It consists of a single brachial cuff attached to a Holter-like device that is made to be worn over a 24-h period. The device has been validated for brachial blood pressure measurement using both the British Hypertension Society and the International Society of Hypertension guidelines [30, 31]. As it uses the same algorithm as the SphygmoCor XCEL, it should provide aortic waveform data with similar accuracy to the SphygmoCor XCEL. 


\section{Advantages and Disadvantages of SphygmoCor Devices}

In the time since AtCor Medical brought SphygmoCor on the market, a large number of companies have established devices that also non-invasively assess certain parameters of aortic pressure and arterial stiffness. The major advantages of some of these devices are their standalone nature (no requirement for coupling to a laptop or personal computer, making them more portable) and their significantly lower price. However, validation, population, and subpopulation thoroughness of data, as well as the clinical utility of these devices, varies. There are more than 1,000 peer-reviewed papers and studies providing data on SphygmoCormeasured parameters in the general population and in specific disease groups, far exceeding publications on all other devices. Additionally, SphygmoCor devices are the only devices cleared by the Food and Drug Administration for non-invasive assessment of the central aortic pressure waveform, making them clinically relevant.

\section{Clinical Evidence and Trials}

SphygmoCor technology has been reported on in the literature every year since 1998. There are 815 peer-reviewed publications that specifically cite SphygmoCor technology (as of March 2016, Scopus, excluding letters [ $n=45]$, reviews [ $n=62]$, conference papers [ $n=$ $41]$, and notes [ $n=23]$ ). Of those, 424 use at least 1 of the arterial stiffness parameters outlined in this article, with the majority using cfPWV (314 articles) or AIx (159 articles). Many of these address large artery stiffness in disease, including but not limited to hypertension [32], renal disease [33], diabetes [34], coronary artery conditions [35, 36], and heart failure [37], as well as postsurgical risk quantification such as in coronary artery intervention [38]. As review articles exist on arterial stiffness in these diseases elsewhere, and a thorough review of all would not be possible here, the remainder of this article addresses the population data gathered on SphygmoCor technology measurement of large artery stiffness, and the clinical use of these values.

\section{Change in Arterial Stiffness with Age}

It is well established that the arteries become stiffer with age [1], even though different arterial regions stiffen at different rates $[5,39]$ and different parameters that correspond to arterial stiffness change at different rates with age [6]. Population normal values based on age and gender are built into the SphygmoCor systems, and the patients' data displayed against the population normal range for the measured stiffness parameters. The data draw on the Anglo-Cardiff Collaborative Trial, which included central aortic pressure waveform measurements such as the AIx from 4,001 healthy individuals (normotensive individuals free from diabetes, high cholesterol, renal disease, and cardiovascular disease) aged 18-90 years as well as cfPWV measurements from a subset of 998 subjects [6]. The SphygmoCor software takes an individual's AIx or cfPWV and reports the mean age of the healthy individuals with that value of arterial stiffness as a secondary guide as to whether an individual's arterial stiffness value lays outside the population norm for healthy individuals.

Other studies including reference values from the general population are the Czech postMONICA study (291 subjects for cfPWV and AIx [40]) and the Copenhagen City Heart study (4,561 subjects for AIx alone [41]). These reference standards from the "general" population are likely to mostly contain Caucasian individuals (Anglo-Cardiff Collaborative Trial arterial stiffness values, 92\% Caucasian; post-MONICA and Copenhagen City Heart study demographics for arterial stiffness studies not published). Reference values for aortic AIx and cfPWV measured using the SphygmoCor system also exist for certain subpopulations, 
including Chinese (924 subjects, cfPWV and AIx [42]), South Africans of South African ancestry (347 subjects, cfPWV and AIx [12]), the Omani Arab population (120 subjects, cfPWV and AIx) [43], and Caucasian Europeans (870 subjects, AIx) [44].

\section{Use of Arterial Stiffness in Clinical Treatment}

A clinical working group sponsored by the North American Artery association recommended that changes in AIx should be considered instead of absolute values with the target being a reduction in AIx [45], indicating a reduced wave reflection magnitude or transit time. However, a reduced AIx may also be caused by an increase in heart rate. In the same manner, antihypertensive therapies that lower heart rate, such as $\beta$-blockers, will increase the absolute value of AIx driven by the heart rate reduction. Unless the heart rate is being taken into account by the operator, it may be more instructive to use a heart rate-normalized AIx such as the AIx at $75 \mathrm{bpm}$ provided by the SphygmoCor software.

A target value for cfPWV of $\leq 10 \mathrm{~m} / \mathrm{s}$ has been recommended by a working group on behalf of the Artery Society, the European Society of Hypertension Working Group on Vascular Structure and Function, and the European Network for Noninvasive Investigation of Large Arteries [46] that has been adopted in the European Society of Hypertension guidelines for hypertension management [47]. This cut-off value uses the newly proposed distance measurement of 0.8 times the direct carotid-to-femoral distance rather than the subtraction technique (suprasternal notch-to-femoral distance minus suprasternal notch-to-carotid distance) that has been used since 1934 [48]. Whilst the 0.8 multiplier provides an estimate of the carotid-femoral path length on average 9.9\% closer to the distance as measured using MRI, this figure of accuracy is highly dependent on the population chosen, since age is a large confounder [11]. The SphygmoCor system default for path length measurement is to use a subtraction of the suprasternal notch-to-carotid measurement from the suprasternal notchto-femoral measurement. This is so as to be consistent with measurements that have been taken with the device from its origin in 1998 as well as with historical measurements of cfPWV before that time, and for comparison to the large population studies that existed before modified distance measurements were proposed.

Like the AIx, a lower cfPWV indicates an improvement in arterial stiffness. Indeed, a reduction in stiffness as indicated by any of the large artery stiffness parameters mentioned previously is a positive result. This may be driven through a decrease in aortic blood pressure, as can be calculated by the SphygmoCor systems, or through geometric or morphological changes in the large arteries.

\section{Prospective View}

With increasing use of ambulatory measurement for waveform parameters associated with arterial stiffness, the application of this to population and disease subgroups is intended. The use of cuff-based acquisition of the pulse has made devices more operator independent, and it is the intention to continue this trend, making the device more user friendly without sacrificing its accuracy and being consistent with the past measurement of arterial stiffness indices. Such measures could include out-of-clinic measurement of parameters like PWV. The conventional measurement of cfPWV is too involved for out-of-clinic - and certainly for ambulatory - measurement. However, alternate methods and correlates may present a way of incorporating this robust measure of arterial stiffness into an ambulatory device. Such measurements would provide a more thorough examination of arterial stiffness in the daily life of a patient, just as ambulatory brachial and aortic blood pressure currently provide a thorough out-of-clinic examination. 
Butlin and Qasem: Large Artery Stiffness Assessment Using SphygmoCor Technology

\section{Disclosure Statement}

The authors have no conflicts of interest to disclose.

\section{References}

1 Butlin M, Avolio AP: Age-related changes in the mechanical properties of arteries; in Derby B, Akhtar R (eds): Mechanical Properties of Aging Soft Tissue. New York, Springer, 2014, pp 37-74.

2 Vlachopoulos C, Aznaouridis K, O’Rourke MF, Safar ME, Baou K, Stefanadis C: Prediction of cardiovascular events and all-cause mortality with central haemodynamics: a systematic review and meta-analysis. Eur Heart J 2010;31:1865-1871.

3 Mancia G, De Backer G, Dominiczak A, Cifkova R, Fagard R, Germano G, etal: 2007 guidelines for the management of arterial hypertension: the task force for the management of arterial hypertension of the European Society of Hypertension (ESH) and of the European Society of Cardiology (ESC). J Hypertens 2007;25:1105-1187.

4 Ben-Shlomo Y, Spears M, Boustred C, May M, Anderson SG, Benjamin EJ, et al: Aortic pulse wave velocity improves cardiovascular event prediction: an individual participant meta-analysis of prospective observational data from 17,635 subjects. J Am Coll Cardiol 2014;63:636-646.

5 Hickson SS, Butlin M, Graves M, Taviani V, Avolio AP, McEniery CM, et al: The relationship of age with regional aortic stiffness and diameter. JACC Cardiovasc Imaging 2010;3:1247-1255.

6 McEniery CM, Yasmin, Hall IR, Qasem A, Wilkinson IB, Cockcroft JR: Normal vascular aging: differential effects on wave reflection and aortic pulse wave velocity: the Anglo-Cardiff Collaborative Trial (ACCT). J Am Coll Cardiol 2005;46:1753-1760.

7 Wilkinson IB, McEniery CM, Schillaci G, Boutouyrie P, Segers P, Donald A, et al: ARTERY Society guidelines for validation of non-invasive haemodynamic measurement devices: part 1, arterial pulse wave velocity. Artery Res 2010;4:34-40.

8 Wilkinson IB, Fuchs SA, Jansen IM, Spratt JC, Murray GD, Cockcroft JR, et al: Reproducibility of pulse wave velocity and augmentation index measured by pulse wave analysis. J Hypertens 1998;16(pt 2):2079-2084.

9 Frimodt-Møller M, Nielsen AH, Kamper A-L, Strandgaard S: Reproducibility of pulse-wave analysis and pulsewave velocity determination in chronic kidney disease. Nephrol Dial Transplant 2008;23:594-600.

10 Weber T, Ammer M, Rammer M, Adji A, O’Rourke MF, Wassertheurer S, et al: Noninvasive determination of carotid-femoral pulse wave velocity depends critically on assessment of travel distance: a comparison with invasive measurement. J Hypertens 2009;27:1624-1630.

11 Huybrechts SAM, Devos DG, Vermeersch SJ, Mahieu D, Achten E, de Backer TLM, et al: Carotid to femoral pulse wave velocity: a comparison of real travelled aortic path lengths determined by MRI and superficial measurements. J Hypertens 2011;29:1577-1582.

12 Shiburi CP, Staessen JA, Maseko M, Wojciechowska W, Thijs L, Van Bortel LM, et al: Reference values for SphygmoCor measurements in South Africans of African ancestry. Am J Hypertens 2006;19:40-46.

13 Avolio AP, Deng FQ, Li WQ, Luo YF, Huang ZD, Xing LF, et al: Effects of aging on arterial distensibility in populations with high and low prevalence of hypertension: comparison between urban and rural communities in China. Circulation 1985;71:202-210.

14 Butlin M, Qasem A, Battista F, Bozec E, McEniery CM, Millet-Amaury E, et al: Carotid-femoral pulse wave velocity assessment using novel cuff-based techniques: comparison with tonometric measurement. J Hypertens 2013;31:2237-2243.

15 Karamanoglu M, O'Rourke MF, Avolio AP, Kelly RP: An analysis of the relationship between central aortic and peripheral upper limb pressure waves in man. Eur Heart J 1993;14:160-167.

16 Wilkinson IB, Mohammad NH, Tyrrell S, Hall IR, Webb DJ, Paul VE, et al: Heart rate dependency of pulse pressure amplification and arterial stiffness. Am J Hypertens 2002;15(pt 1):24-30.

17 Takazawa K, O’Rourke MF, Fujita M, Tanaka N, Takeda K, Kurosu F, et al: Estimation of ascending aortic pressure from radial arterial pressure using a generalised transfer function. Z Kardiol 1996;85(suppl 3):137-139.

18 Chen CH, Nevo E, Fetics B, Pak PH, Yin FC, Maughan WL, et al: Estimation of central aortic pressure waveform by mathematical transformation of radial tonometry pressure. Validation of generalized transfer function. Circulation 1997;95:1827-1836.

19 Söderström S, Nyberg G, O’Rourke MF, Sellgren J, Pontén J: Can a clinically useful aortic pressure wave be derived from a radial pressure wave? Br J Anaesth 2002;88:481-488.

20 O'Rourke MF, Pauca AL: Augmentation of the aortic and central arterial pressure waveform. Blood Press Monit 2004;9:179-185.

21 Hwang MH, Yoo JK, Kim HK, Hwang CL, Mackay K, Hemstreet O, et al: Validity and reliability of aortic pulse wave velocity and augmentation index determined by the new cuff-based SphygmoCor Xcel. J Hum Hypertens 2014;28:475-481.

22 Filipovský J, Svobodová V, Pecen L: Reproducibility of radial pulse wave analysis in healthy subjects. J Hypertens 2000;18:1033-1040.

23 Siebenhofer A, Kemp C, Sutton A, Williams B: The reproducibility of central aortic blood pressure measurements in healthy subjects using applanation tonometry and sphygmocardiography. J Hum Hypertens 1999; $13: 625-629$. 
24 Papaioannou TG, Stamatelopoulos KS, Gialafos E, Vlachopoulos C, Karatzis E, Nanas J, et al: Monitoring of arterial stiffness indices by applanation tonometry and pulse wave analysis: reproducibility at low blood pressures. J Clin Monit Comput 2004;18:137-144.

25 Savage MT, Ferro CJ, Pinder SJ, Tomson CRV: Reproducibility of derived central arterial waveforms in patients with chronic renal failure. Clin Sci (Lond) 2002;103:59-65.

26 Westerhof N, Sipkema P, van den Bos GC, Elzinga G: Forward and backward waves in the arterial system. Cardiovasc Res 1972;6:648-656.

27 Westerhof BE, Guelen I, Westerh of N, Karemaker JM, Avolio A: Quantification of wave reflection in the human aorta from pressure alone: a proof of principle. Hypertension 2006;48:595-601.

28 Qasem A, Avolio AP: Determination of aortic pulse wave velocity from waveform decomposition of the central aortic pressure pulse. Hypertension 2008;51:188-195.

29 Crilly M, Coch C, Bruce M, Clark H, Williams D: Indices of cardiovascular function derived from peripheral pulse wave analysis using radial applanation tonometry: a measurement repeatability study. Vasc Med 2007;12: 189-197.

30 Goodwin J, Bilous M, Winship S, Finn P, Jones SC: Validation of the Oscar 2 oscillometric 24-h ambulatory blood pressure monitor according to the British Hypertension Society protocol. Blood Press Monit 2007;12:113117.

31 Jones SC, Bilous M, Winship S, Finn P, Goodwin J: Validation of the OSCAR 2 oscillometric 24-hour ambulatory blood pressure monitor according to the International Protocol for the validation of blood pressure measuring devices. Blood Press Monit 2004;9:219-223.

32 Gedikli O, Ozturk S, Yilmaz H, Baykan M, Kiris A, Durmus I, et al: Relationship between arterial stiffness and myocardial damage in patients with newly diagnosed essential hypertension. Am J Hypertens 2008;21:989993.

33 Rossi SH, McQuarrie EP, Miller WH, Mackenzie RM, Dymott JA, Moreno MU, et al: Impaired renal function impacts negatively on vascular stiffness in patients with coronary artery disease. BMC Nephrol 2013;14:173.

34 Cruickshank K, Riste L, Anderson SG, Wright JS, Dunn G, Gosling RG: Aortic pulse-wave velocity and its relationship to mortality in diabetes and glucose intolerance: an integrated index of vascular function? Circulation 2002;106:2085-2090.

35 Chirinos JA, Zambrano JP, Chakko S, Veerani A, Schob A, Willens HJ, et al: Aortic pressure augmentation predicts adverse cardiovascular events in patients with established coronary artery disease. Hypertension 2005;45:980-985.

36 Nichols WW, Denardo SJ, Davidson JB, Huo T, Bairey Merz CN, Pepine CJ: Association of aortic stiffness and wave reflections with coronary flow reserve in women without obstructive coronary artery disease: an ancillary study from the National Heart, Lung, and Blood Institute-sponsored Women's Ischemia Syndrome Evaluation (WISE). Am J Heart;170:1243-1254.

37 Weber T, Auer J, Lamm G, O’Rourke MF, Eber B: Arterial stiffness, central blood pressures, and wave reflections in cardiomyopathy - implications for risk stratification. J Card Fail 2007;13:353-359.

38 Weber T, Auer J, O’Rourke MF, Kvas E, Lassnig E, Lamm G, et al: Increased arterial wave reflections predict severe cardiovascular events in patients undergoing percutaneous coronary interventions. Eur Heart J 2005; 26:2657-2663

39 Avolio AP, Chen SG, Wang RP, Zhang CL, Li MF, O'Rourke MF: Effects of aging on changing arterial compliance and left ventricular load in a northern Chinese urban community. Circulation 1983;68:50-58.

40 Filipovský J, Tichá M, Cífková R, Lánská V, Stastná V, Roucka P: Large artery stiffness and pulse wave reflection: results of a population-based study. Blood Press 2005;14:45-52.

41 Janner JH, Godtfredsen NS, Ladelund S, Vestbo J, Prescott E: Aortic augmentation index: reference values in a large unselected population by means of the SphygmoCor device. Am J Hypertens 2010;23:180-185.

42 Li Y, Staessen JA, Li LH, Huang QF, Lu L, Wang JG: Reference values for the arterial pulse wave in Chinese. Am J Hypertens 2008;21:668-673.

$43 \mathrm{Al}$-Hashmi KM, Al-Abri MA, Al-Zakwani IS, Al-Waili KA, Al-Rasadi KH, Al-Sabti HA, et al: Reference values for arterial stiffness indices in an Omani Arab population. Saudi Med J 2014;35:1367-1372.

44 Wojciechowska W, Staessen JA, Nawrot T, Cwynar M, Seidlerová J, Stolarz K, et al: Reference values in white Europeans for the arterial pulse wave recorded by means of the SphygmoCor device. Hypertens Res 2006;29: 475-483.

45 Townsend RR, Black HR, Chirinos JA, Feig PU, Ferdinand KC, Germain M, et al: Clinical use of pulse wave analysis: proceedings from a symposium sponsored by North American Artery. J Clin Hypertens (Greenwich) 2015;17:503-513.

46 Van Bortel LM, Laurent S, Boutouyrie P, Chowienczyk P, Cruickshank JK, De Backer T, et al: Expert consensus document on the measurement of aortic stiffness in daily practice using carotid-femoral pulse wave velocity. J Hypertens 2012;30:445-448.

47 Mancia G, Fagard R, Narkiewicz K, Redon J, Zanchetti A, Böhm M, et al: 2013 ESH/ESC guidelines for the management of arterial hypertension: the Task Force for the Management of Arterial Hypertension of the European Society of Hypertension (ESH) and of the European Society of Cardiology (ESC). Eur Heart J 2013; 34:2159-2219.

48 Hallock P: Arterial elasticity in man in relation to age as evaluated by the pulse wave velocity method. Arch Intern Med 1934;54:770-798. 